\title{
Semiclassics of Andreev billiards
}

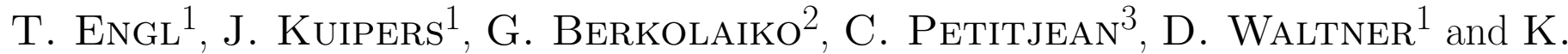 RICHTER ${ }^{1}$}

${ }^{1}$ Institute for Theoretical Physics, University of Regensburg, 93040 Regensburg, Germany

${ }^{2}$ Department of Mathematics, Texas A\&M University, College Station, TX 77843-3368, USA

${ }^{3}$ SPSMS, UMR-E 9001, CEA-INAC/UJF-Grenoble 1, 17 Rue des Martyrs, 38054 Grenoble Cedex 9, France 


\section{Semiclassics of Andreev billiards}

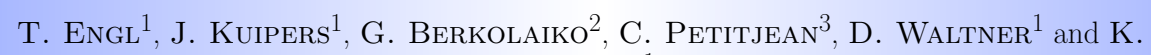
RICHTER $^{1}$

${ }^{1}$ Institute for Theoretical Physics, University of Regensburg, 93040 Regensburg, Germany

2 Department of Mathematics, Texas A\&M University, College Station, TX 77843-3368, USA

${ }^{3}$ SPSMS, UMR-E 9001, CEA-INAC/UJF-Grenoble 1, 17 Rue des Martyrs, 38054 Grenoble Cedex 9, France

\section{By coupling a superconductor to a quantum cavity or dot one may decide}
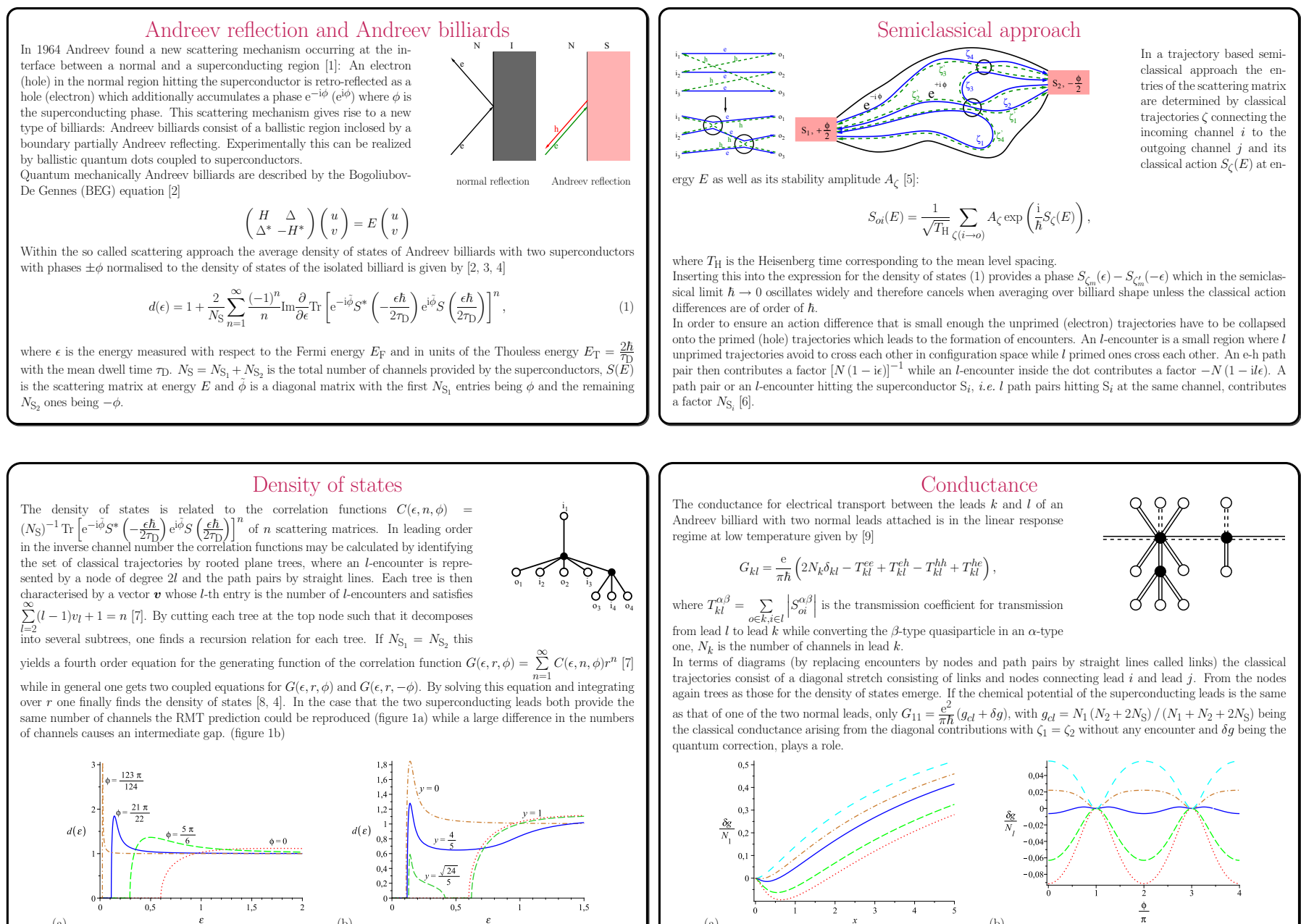

\section{Density of states}

The density of states to the correlation functions $C(\epsilon, n, \phi)=$

of $n$ scattering matrices. In leading orde

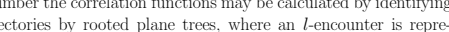

ented by a node of degree $2 l$ and the path pairs by straight lines. Each tree is the

each tree at the top node such that it decompose

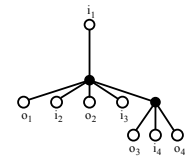

$\mathrm{O}_{3} \mathrm{O}_{4} \mathrm{O}_{4}$

Figure 1: Density of states of an Andreev billiard with two superconducting leads with (a) equal numbers of channels at different phase differences and with (b) $y=\left(N_{\mathrm{S}_{1}}-N_{\mathrm{S}_{2}}\right) / N_{\mathrm{S}}$ at phase difference $\phi=21 \pi / 22$. If the phase difference

is large enough, by increasing the difference between the numbers of channels a intermediate gap may occur.

\section{Conductance} The conductance for electrical transport between the leads $k$ and $l$ of an
Andreev billiard with two normal leads attached is in the linear response regime at low temperature given by $[9]$

$$
G_{k l}=\frac{\mathrm{e}}{\pi \hbar}\left(2 N_{k} \delta_{k l}-T_{k l}^{e e}+T_{k l}^{e h}-T_{k l}^{h h}+T_{k l}^{h e}\right),
$$

where $T_{k l}^{\alpha \beta}=\sum_{j=1}\left|S_{o i}^{\alpha \beta}\right|$ is the transmission coefficient for transmission

from lead $l$ to lead $k$ while converting the $\beta$-type quasiparticle in an $\alpha$-type

one, $N_{k}$ is the number of channels in lead $k$.

In terms of diagrams (by replacing encounters by nodes and path pairs by straight lines called links) the classical trajectories consist of a diagonal stretch consisting of links and nodes connecting lead $i$ and lead $j$. From the nodes again trees as those for the density of states emerge. If the chemical potential of the superconducting leads is the same as that of one of the two normal leads, only $G_{11}=\frac{\mathrm{e}^{2}}{\pi \hbar}\left(g_{c l}+\delta g\right)$, with $g_{c l}=N_{1}\left(N_{2}+2 N_{\mathrm{S}}\right) /\left(N_{1}+N_{2}+2 N_{\mathrm{S}}\right)$ being
the classical conductance arising from the diagonal contributions with $\zeta_{1}=\zeta_{2}$ without any encounter and $\delta g$ being the the classical conductance arising
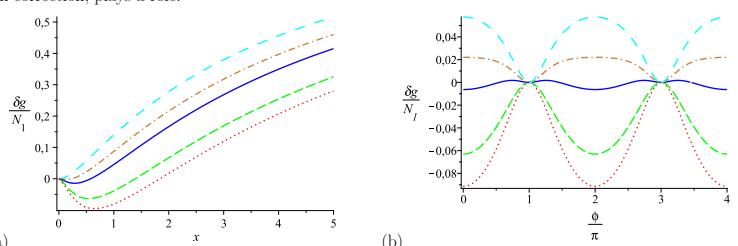
Figure 2: Conductance correction for $N_{2} / N_{1} \rightarrow 0$ (dotted), $N_{2} / N_{1}=0.2$ (dashed), $N_{2} / N_{1}=1$ (solid), $N_{2} / N_{1}=2$
(dash dotted) and $N_{2} / N_{1}=7$ (space dashed) as a function of (a) $x=N_{\mathrm{S}}$ ( $\left(N_{1}+N_{2}\right.$ ) at $\phi=0$ and (b) as a function of the phase difference $\phi$ at $x=0.5$.
Conclusion and Outlook

Semiclassics based on the diagonal contribution is not sufficient to explain Andreev billiards. In fact correlations between several orbits are important to describe the effects of superconductors in proximity to a quantum do

The results for the conductance show that the effect of the superconductor is of the order of the numbers of channels and may therefore be very important. The phase difference between the superconductors may also play a crucial role

Note that the results presented here are only valid for large total numbers of channels $N=N_{1}+N_{2}+N_{\mathrm{S}}$ but each specific number of channels $N_{1}, N_{2}, N_{\mathrm{S}_{1}}$ $\mathrm{N}_{\mathrm{S}_{2}}$ may be small. This however means that e.g. weak localisation corrections have not been included yet.

$g$ order in the ratio $N_{\mathrm{S}} /\left(N_{1}+N_{2}\right)$ in $[10]$ as of the conductance non-zero temperature has also been

urther possible investigations would be the shot noise of Andreev billiards or the effect of tunnel barriers

\section{References}

[1] A. F. ANDREEV, Sov. Phys. JETP 19, 1228 (1964).

[2] C. W. J. BeEnakker, Lect. Notes. Phys. 667, 131 (2005).

[3] E. Doron and U. SMiLANSKY, Phys. Rev. Lett. 68, 1255 (1992).

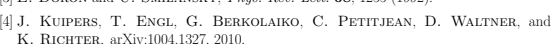

[5] W. H. MLLLER, Adv. Chem. Phys 30, 77 (1975).

[6] S. MÜLLER, S. Heusler, P. Braun, and F. HaAkE, New J. Phys. 9 , 12 (2007)

[7] G. Berkolaiko, J. M. Harrison, and M. Novaes, J. Phys. A 41,365102 (2008

[8] J. Kuipers, D. Waltner, C. PettTjean, G. Berkolaiko, and K. Richter

C. J. Lambert and R. Raimond. $J$ Phys. Cond. Mat. 10.901 (1998)

10] P. JACQUOD and R. S. WhITNEY, arXiv:0910.2943, 2009 